\title{
La aceleración en la lógica del capital ${ }^{*}$ \\ Acceleration in the Logic of Capital \\ A aceleração na lógica do capital
}

Javier Luis Cristiano a
Universidad Nacional de Córdoba, Argentina
javier.cristiano.m@gmail.com
ORCID: https://orcid.org/0000-0001-5731-7269

DOI: https://doi.org/10.11144/Javeriana.uh88.lalc

Recibido: 18 Julio 2017

Aceptado: 28 Mayo 2019

Publicado: 20 Diciembre 2019

\begin{abstract}
Resumen:
El trabajo retoma la argumentación de Hartmut Rosa sobre la aceleración como principio tendencial de la modernidad, ahondando en las razones (los "motores") que producen dicho proceso. Uno de esos motores, el que aquí se analiza en detalle, es el de la competencia capitalista, que impulsa junto con aspectos culturales e institucionales el incremento de la velocidad. Apelando a la obra de David Harvey se muestra en qué sentidos y por qué razones lógicas el capitalismo produce, en efecto, aceleración, lo que permite luego realizar dos contribuciones a la teorización de Rosa: discutir la relación entre los distintos motores de la aceleración y sopesar la importancia de considerarlos en términos de un concepto sociológico de estructura social.
\end{abstract}

Palabras clave: estructura, tiempo, aceleración, capitalismo.

Abstract:

This work resume the arguments by Hartmut Rosa on the acceleration as a trendy principle of modernity, thus studying in depth the reasons (the "drives") leading to this process. One of these drives -the one analyzed herein in detail- is the capitalist competition that fuels, added to other cultural and institutional aspects, the increase in the speed. Based on the works by David Harvey, this paper shows the directions and the logic reasons whereby the capitalism actually produces the acceleration. Then, it allows making two contributions to Rosa's theory: a discussion of the relationship between the different drives of acceleration; and a weighing of how important it is to take them under the sociological concept of social structure.

Keywords: structure, time, acceleration, capitalism.

\section{Resumo:}

O trabalho retoma a argumentação de Hartmut Rosa sobre o aceleramento como princípio tendencial da modernidade, aprofundando-se nas razoes (os "motores") que produzem esse processo. Um desses motores, aqui analisado em detalhe, é o da competência capitalista, que impulsiona junto com aspectos culturais e institucionais o acréscimo da velocidade. Recorrendo à obra de David Harvey, mostra-se em quais sentidos e por quais razoes lógicas o capitalismo produz de fato, aceleração, o que possibilita após realizar duas contribuições à teorização de Rosa: discutir a relação entre os diferentes motores da aceleração e ponderar a importância de considera-los em termos de um conceito sociológico de estrutura social.

Palavras-chave: estrutura, tempo, aceleração, capitalismo.

\section{El régimen acelerador totalitario: la tesis de Hartmut Rosa}

En sus trabajos de los últimos quince años Hartmut Rosa ha logrado afirmar en el campo del pensamiento crítico una teoría de la aceleración ${ }^{1}$ según la cual la velocidad creciente de los procesos sociales resulta una característica dominante de la modernidad tardía. Rosa no duda en ubicar su análisis en un horizonte valorativo, pues considera que la aceleración es una nueva cara de la alienación. En sus palabras, nos encontramos "[...] férreamente regulados, dominados y reprimidos por un régimen temporal en gran parte

Notas de autor

\footnotetext{
a Autor de correspondencia. Correo electrónico: javier.cristiano.m@gmail.com
} 
invisible, despolitizado, no discutido, subteorizado y no articulado" (Rosa, 2016, p. 10), que precisamente por eso merece llamarse totalitario.

Las razones por las que debiéramos combatirlo son muchas y van desde cuestiones subjetivas y psicológicas (la velocidad produce saturación del yo, genera estructuras psíquicas de culpa, etcétera) hasta cuestiones macrosociales, como la desincronización que experimenta el sistema político respecto al económico o al tecnológico. Pero sobre todo se trata de la ruptura de la promesa básica de la modernidad, según la cual las pautas de la vida individual y colectiva deberían definirse autónoma y reflexivamente. "El tiempo, hasta ahora, se encuentra más allá del alcance de la política" (Rosa, 2016, p. 110), lo que significa que el régimen de aceleración es inconsciente, ajeno a cualquier decisión vinculante y, en consecuencia, carente de legitimidad política.

El punto que nos interesa específicamente de su argumento es el de los "motores" que producen la aceleración social. Con mínimas variantes en sus distintos trabajos (Rosa, 2013, pp. 160-194; 2011, pp. 20-26), Rosa identifica tres:

1. El capitalismo y la competencia es el motor principal en la medida en que la velocidad representa una ventaja competitiva y la competencia impregna el conjunto de la vida social como mecanismo dominante de asignación de recursos.

2. Hay luego un factor cultural al que Rosa denomina "promesa de eternidad". Su razonamiento es que en las sociedades seculares, que han expandido hasta el límite la oferta de experiencias y opciones de todo tipo, la aceleración funciona como equivalente funcional de la promesa religiosa de la vida eterna, pues hacer más cosas en menos tiempo es la respuesta lógica a un sobreestímulo de opciones que no pueden abarcarse en el tiempo de una vida.

3. La propulsión mutua de tres fenómenos: el desarrollo tecnológico, el aumento de la tasa de cambio social y el incremento del ritmo de vida. Con lo primero se refiere Rosa a los medios técnicos que aceleran procesos racionales con arreglo a fines; con lo segundo, a la inestabilidad de los valores, las instituciones y las creencias de todo tipo; con lo tercero, al incremento del número de episodios de experiencia y acción por unidad de tiempo. Los tres se impulsan mutualmente: la aceleración tecnológica desplaza marcos institucionales y normativos, pero al mismo tiempo ensancha el espectro de opciones y la necesidad consiguiente de tiempo; el cambio social impone ritmos crecientes al desarrollo tecnológico y estira las exigencias del ritmo de vida, etcétera.

La metáfora de los motores conduce el argumento de Rosa hacia una teoría sustantiva de la sociedad, pues se supone que son las fuerzas estructurales que explican la alienación dromológica. Esto pone en el centro la cuestión de la naturaleza y el funcionamiento de cada motor, amén de las relaciones que mantienen entre sí. En lo que sigue vamos a centrar nuestra atención en el motor que Rosa considera primordial, el capitalismo y la competencia, con la intención de precisar por qué y de qué modo produce en efecto aceleración social. Para eso vamos a apelar a la obra de David Harvey, una de las pocas que se ha ocupado de los aspectos temporales en el análisis crítico de la economía capitalista. ${ }^{2}$ Después de hacerlo volveremos al argumento de Rosa para sacar el partido que creemos puede extraerse de nuestro análisis: revisar las relaciones entre los distintos motores y abrir la discusión sobre el interés de considerarlos factores estructurales del cambio temporal. La contribución de nuestro trabajo consiste por lo tanto en reforzar críticamente la sociología de la aceleración de Rosa.

Antes de empezar, aclaramos el sentido que da el autor al término "aceleración", que en lo esencial tiene dos componentes: el aumento del número de episodios de acción por unidad de tiempo, que mencionamos recién, y la pérdida de confianza en las experiencias previas y en la certeza de las expectativas hacia el futuro. Lo primero hace referencia a la tendencia a hacer más cosas en menos tiempo, a comprimir el horizonte temporal de la acción y la decisión; lo segundo, a una suerte de presentificación, que implica "la contracción de los lapsos de tiempo disponibles como presente" (Rosa, 2013, p. 26). Acelerar, por tanto, es hacer y experimentar más en menos tiempo y estrechar el presente como dimensión temporal en detrimento del pasado y del futuro. 


\section{El valor: la velocidad en el núcleo estructural del capitalismo}

El primer paso de nuestro análisis tiene que ser una precisión básica sobre la naturaleza del capitalismo. En la lectura que Harvey hace de Marx, tres son sus rasgos principales:

1. El capitalismo es un orden económico que transforma la lógica mercancía-dinero-mercancía $(\mathrm{MDM})$ en la lógica dinero-mercancía-dinero (DMD), que implica la definición del capital como "dinero puesto en circulación para obtener más dinero" (Harvey, 1991, pp. 24-25). Lo que denominamos "lógica del capital", o indistintamente "proceso de valorización" o "lógica de la expansión del valor", alude a ese proceso que empieza en la propiedad privada de dinero y termina en una propiedad mayor, pasando por el proceso de producción y circulación de mercancías.

2. En su contenido más concreto, el proceso DMD tiene en el capitalismo la siguiente forma: 1 . D -- $[\mathrm{FT}(\mathrm{cv})+\mathrm{MP}(\mathrm{cf})]$--- M ---- D'

que significa: el dueño del capital compra fuerza de trabajo (capital variable) y medios de producción (capital fijo) para producir una mercancía $(\mathrm{M})$ que se vende en el mercado para reconvertirla en dinero + ganancias (D’). Esto significa que la relación salarial, y por ende la explotación del trabajo, da en el capitalismo el contenido específico al proceso DMD.

3. En tercer lugar, lo propio del capitalismo es la concurrencia en un mercado formalmente libre para la compra/venta de fuerza de trabajo y capital fijo, y para la compra/venta de los bienes producidos.

Sobre esta base podemos identificar rápidamente dos aspectos en que la aceleración es una tendencia estructural del capitalismo:

1. En la medida en que el valor de una mercancía depende del tiempo de trabajo empleado para producirla, el tiempo se ubica en el núcleo de la producción capitalista. Es sabido que Marx toma de Ricardo la idea de que lo que tienen en común todas las mercancías es el hecho de ser productos del trabajo, lo que permite su intercambio en el mercado. Marx da a este principio un giro fundamental al considerar que no se trata del tiempo en general sino del tiempo "social medio necesario" para producir una mercancía, en el estado de las habilidades y destrezas medias del momento, con su grado de desarrollo tecnológico y organizativo correspondiente. Esto es lo que permite resolver la paradoja de que si lo que se intercambia en el mercado son tiempos de trabajo relativos, convendría a cada productor trabajar más lento para que su trabajo se valorice más. En la medida en que se trata de una magnitud promedio, no solo es necesario alcanzar el ritmo común sino que se vuelve importante aumentarlo. Ese incremento puede llamarse aceleración porque implica un proceso en espiral: en la medida en que un capitalista logre producir por debajo del tiempo medio, la tendencia será a que los demás alcancen la misma meta, con lo que el promedio se moverá hacia arriba, volviendo el ciclo a empezar.

La aceleración, por tanto, es la consecuencia lógica del capitalismo analizado desde el punto de vista del valor, más concretamente, la aceleración de los tiempos de producción de las mercancías, que en el orden capitalista se producen al solo efecto de ser vendidas en el mercado. ${ }^{3}$

2. También hay aceleración en la lógica más amplia del proceso que describe la fórmula entera (D -$[\mathrm{FT}(\mathrm{cv})+\mathrm{MP}(\mathrm{cf})]$--- M ---- D'). Harvey retoma aquí de Marx el concepto de tiempo de rotación, que es el tiempo que tarda el capital en recorrer todo el circuito. Cuando más veces haga el capital la vuelta completa mayor será la tasa de ganancia. Así, por ejemplo, el fabricante de indumentaria que consiga fabricar y vender más cantidad de veces en un año tendrá un beneficio promedio mayor que sus competidores en ese rubro. En otros puede que se gane más en términos nominales, pero una vez situado en uno, la tasa de ganancia depende de la cantidad de veces que pueda completar el proceso. 
Acelerar los tiempos de rotación constituye, en consecuencia, también una presión estructural del capitalismo, de ahí que, según Harvey, los bienes con menor tiempo de rotación atraigan tendencialmente la inversión, y de ahí también la tendencia del capitalismo a lo que Harvey denomina "contracción espacio-temporal" (Harvey, 1999, pp. 267-287). Para que el ciclo de rotación se reduzca, se debe disminuir todo lo posible la distancia espacial y temporal entre cada paso del proceso; por ejemplo, se debe expandir todo lo posible el ámbito de circulación (el mercado), se debe hacer todo lo dinámico que se pueda el movimiento de capital (para pasar, por ejemplo, de una rama de la producción a otra), se debe vender lo más rápido posible, tener disposición inmediata de la fuerza de trabajo, etcétera. Una notable frase de Marx, citada reiteradamente por Harvey, da cuenta de esta tendencia: el capitalismo aspira "[...] al paso del capital de una fase a otra con la misma velocidad del pensamiento" (Marx, Grundrisse, citado en Harvey, 1999, p. 95).

\section{Las crisis periódicas como crisis de lentitud}

Las anteriores son tendencias internas a la lógica del capital, no algo impuesto desde afuera, pero también son estructurales las crisis periódicas, tan decisivas en el proyecto político asociado a Marx, pero sobre todo en los análisis posteriores de las tendencias del capitalismo a gran escala. Uno de los aportes principales de Harvey ha sido dar a la teoría marxiana de la crisis una forma teórica consistente, ordenando materiales dispersos de la obra y desplegando, en trabajos posteriores, esos análisis sobre procesos históricos específicos (Harvey, 2007, 2014). No podemos abordar en detalle las relaciones entre crisis periódicas y aceleración, por lo que nos limitamos al plano definicional en el que las crisis pueden considerarse precisamente desaceleraciones de la producción y de la circulación del capital. En otras palabras, las crisis periódicas son de ralentización, lo que indica por contraste la primacía de la aceleración en el funcionamiento "virtuoso" del capitalismo.

Dos principios hay que fijar para dar contenido a este argumento: uno, que el capital es un proceso más que una cosa (Harvey, 1991, p. 31; 2014, pp. 81-88), más concretamente, el proceso que lleva al capital a través de cada una de sus fases, siguiendo la fórmula extensa de la segunda sección de este artículo. Así, el capital adopta la forma de dinero en el primer momento (D), luego la forma de proceso productivo, después la forma mercancía y finalmente la forma dinero (D’), que permite reiniciar el ciclo. La segunda consideración, vital para nuestro análisis, es que "el capital sigue siendo capital solo como valor en movimiento" (Harvey, 1991, p. 81), pues una cosa es el dinero, como valor acumulado que se puede emplear en diversos usos, y otra es el capital, que consiste en movimiento orientado a su expansión. La crisis supone, por lo tanto, la detención del movimiento en su forma más típica debido a la imposibilidad de realizar el valor en el consumo. Esto ocurre cuando el propio despliegue del capitalismo, que produce acumulación en un polo y desposesión en el otro, lleva a producir más de lo que puede venderse en el mercado. La sobreproducción adopta entonces formas empíricas como capital dinero ocioso, capacidad productiva no utilizada, fuerza de trabajo desempleada o excedente de mercancías en stock. Cada uno de esos fenómenos puede interpretarse en sentido temporal como tardanza, es decir, como desaceleración.

\section{El capital financiero como lubricante temporal}

Las crisis de sobreacumulación corresponden a lo que Harvey denomina el "primer corte" de la teoría de la crisis. Le llama así porque, tratándose de un tema complejo y escasamente saldado, de lo que se trata es de hacer escisiones sucesivas en los procesos de crisis, cortes que permiten ver una parte de su anatomía total. El segundo corte se refiere al tipo de crisis al que estamos habituados en el capitalismo actual: la financiera. Escuetamente, el razonamiento es que el capitalismo hubiese sucumbido rápidamente si no hubiese 
desarrollado un sistema de crédito que modere y aplace sus contradicciones, pero, al mismo tiempo, su desarrollo desproporcionado y autónomo es la base de un tipo específico de crisis. Proponemos que ambos aspectos del capital financiero tienen una dimensión temporal y dan cuenta, cada uno, de aspectos puntuales del proceso de aceleración social.

1. El sistema de crédito tal como lo conocemos es una consecuencia del proceso capitalista de acumulación. Tiene antecedentes en formas económicas anteriores, pero recién con el volumen de la acumulación capitalista surge en su dimensión y en su forma actual. En su sentido más simple, consiste en que un capitalista presta dinero a otro capitalista en la expectativa de recuperarlo luego con un interés. Esto implica un desdoblamiento de las funciones del capital que pone de un lado al capital productivo y del otro al capital financiero, y extrae al final una porción de plusvalía que vale como interés.

Desde el punto de vista temporal este proceso tiene una doble implicancia. Extiende hacia delante la lógica aceleradora primaria (segunda sección de este artículo), disciplinando la producción y su velocidad relativa en una escala de tiempo más amplia. Por otro lado, permite una serie de arreglos temporales que defienden potencialmente al sistema de sus desajustes. El crédito puede financiar la producción pero también trasladar el capital de ramas con tiempos cortos de rotación a ramas con rotaciones largas; permite financiar el consumo, extendiendo hacia delante el problema de exceso de stock; es también el crédito el que permite distribuir en el tiempo los costos de infraestructura física que necesita el capital (caminos, energía). En los dos planos puede usarse la metáfora de la lubricación temporal: lo que en la producción capitalista strictu sensu son tiempos rígidos dados por los procesos materiales de producción, mediante el capital financiero se vuelven tiempos maleables y ajustables a los distintos intereses del capital.

2. En su otra cara el capital financiero produce sus propias crisis, en la medida en que es tanto el salvador del capital como "la matriz de todas sus formas absurdas" (Marx, El capital, citado en Harvey, 1991, p. 273). Lo absurdo surge de la autonomización que produce el juego de espejos de lo que se denomina "capital ficticio" (papeles que representan el valor de otros papeles que representan el valor de otros que representan dinero que representa, a su vez, el valor propiamente dicho, las mercancías en su existencia física). Ese juego tiene como consecuencia una distorsión temporal, pues frente a los tiempos reales de la producción genera tanto la ficción de una ralentización - por ejemplo, posterga artificialmente promesas de pago- como el vértigo que apreciamos en las crisis financieras. En este punto, que es el que nos interesa destacar, la lógica de la acumulación y la destrucción del capital adquieren una velocidad por completo ajena a los parámetros de las mercancías reales y del dinero. Se puede ganar una fortuna en segundos, lo mismo que perderla, lo que es el paroxismo de la aceleración capitalista.

\section{Lo sólido que se desvanece}

Las crisis producen, por supuesto, consecuencias sobre personas y entornos concretos, pero también lo hace el funcionamiento normal del orden capitalista. La expresión de lo sólido que se desvanece en el aire hace referencia también a la aceleración, en este caso la que corresponde al cambio de las coordenadas de vida y sociabilidad que surgen como consecuencia del despliegue del capital.

La primera de esas consecuencias es la que refiere más directamente la metáfora de lo sólido que se desvanece. A lo que se refiere Marx (1988, p. 46) es al carácter revolucionario del capitalismo respecto a las formas de vida tradicionales, que resultan arrasadas por la lógica expansiva del capital. Esa lógica tiene razones precisas: en la medida en que cada capitalista tiene incentivo para pagar lo menos posible, el resultado agregado es un déficit de demanda que está en la base de las crisis de sobreacumulación. La solución suele 
ser la expansión de las fronteras geográficas del capitalismo en la búsqueda de nuevos ámbitos de inversión y nuevos consumidores, proceso en el cual, dice Marx,

\begin{abstract}
el capital fomenta una tendencia a remontarse por sobre las barreras y los prejuicios nacionales y a sobreponerse a la deificación de la naturaleza y al modo tradicional de satisfacer las necesidades, circunscripta dentro de determinados límites concretada a las necesidades existentes y a la reproducción del viejo modo de vida. El capital actúa destructivamente contra todo esto y [ejerce una acción] constantemente revolucionaria, derribando todos los límites que obstruyen el desarrollo de las fuerzas productivas y se oponen a la expansión de las necesidades, a la diversificación de la producción, y a la explotación y el intercambio [libre] de las fuerzas materiales y espirituales. (Grundrisse, citado en Harvey, 1991, p. 102)
\end{abstract}

Por las mismas razones, en segundo lugar, el capital es una máquina de fabricar consumidores. Tan esencial es la producción de nuevos consumos como la explotación de fuerza de trabajo, pues es el consumo lo que completa el ciclo de la fórmula extensa (... M --- D'). Históricamente esto se ha reflejado en el aumento de la cantidad de bienes consumidos y en la ampliación del número de consumidores, pero sobre todo en la modificación de las pautas de consumo, con su consiguiente reestructuración de deseos y necesidades. La relación de estos fenómenos con la aceleración es directa: objetos que duran menos (obsolescencia planificada) y deseos/necesidades también de menor duración tanto en su contenido como en el tiempo que dura la satisfacción.

En tercer lugar, tenemos el desarrollo tecnológico. En la segunda sección, cuando analizamos el vínculo entre el valor y la aceleración, dejamos en suspenso la distinción clásica entre plusvalía absoluta y plusvalía relativa. La primera se refiere a la explotación del trabajo en su sentido lato: el capitalista está obligado a explotar a sus trabajadores al menos hasta alcanzar el nivel correspondiente al tiempo medio que fija el valor de las mercancías; tiene además el incentivo de ir un poco más allá, haciendo idealmente, como dice Marx, que una jornada se convierta en dos. Pero también puede lograrlo innovando tecnológicamente tanto en la técnica en sentido restringido, como en las formas de organización de la producción. Innovar técnicamente es por tanto una consecuencia del proceso capitalista en su forma de plusvalía relativa, lo que explica el extraordinario despliegue tecnológico del capitalismo a lo largo de su historia.

Las implicancias temporales de este proceso son amplias, y de ellas dan cuenta las discusiones actuales sobre la relación entre técnica y experiencia temporal. ${ }^{4}$ Para nuestro argumento podemos limitarnos a tres conexiones con la aceleración social. La más directa es que en la medida en que la tecnología produce plusvalía relativa produce también aceleración —en concreto, aquí, de los tiempos de producción y de rotación-. La segunda es que la tecnología, en tanto conjunto de ramas de la propia producción capitalista, adquiere autonomía y reproduce en su interior la lógica primaria de la aceleración. Puesto que no se trata de un ámbito más, sino de uno sensible para las otras ramas de la producción, sus repercusiones son crecientemente importantes sobre todas ellas. Tercero, las mismas tecnologías penetran en esferas no económicas de la vida social promoviendo efectos sísmicos, como ocurrió en su momento con los medios de comunicación respecto a la vida política, y como sucede hoy con las redes, la informática y el mundo digital. En resumidas cuentas, la lógica del capital da impulso al despliegue tecnológico que adquiere fuerza e intensidad propia, y acelera otros muchos procesos sociales.

En cuarto lugar, tenemos lo que Marx describió como "descapacitación" de los trabajadores. A diferencia de modos de organización anteriores, el trabajo adopta en el capitalismo la forma de "trabajo abstracto", que no vale por su cualidad sino por su cantidad. El paso del trabajo artesanal al industrial, repetitivo, mecánico e independiente de las habilidades es un rasgo distintivo del capitalismo y de la alienación que produce en el trabajador. Las largas discusiones sobre el impacto que esto tiene sobre la identidad y la autorreferencia del trabajador, ${ }^{5}$ y sobre las implicancias de los sucesivos cambios en la forma organizativa del capitalismo, que a veces parece que acrecientan y otras veces que moderan la tendencia general, ${ }^{6}$ no pone en discusión el carácter constantemente cambiante de las habilidades y las disposiciones subjetivas que la lógica del capital impone sobre el trabajador. La tendencia a cambiar de trabajo cada vez más veces en las últimas generaciones, la experiencia del desempleo vivida cada vez por más trabajadores, la obsolescencia más rápida de 
las habilidades y currículums, la lógica vertiginosa de las fusiones y otras alquimias empresariales son algunos de los fenómenos que van en esta dirección.

\section{La lógica del capital como metástasis}

Hasta aquí mostramos algunas razones por las que la lógica del capital empuja procesos de aceleración social. Resumiendo, es una lógica que exige aceleración del tiempo de trabajo en la medida en que el valor está determinado por el tiempo de producción; es un sistema que requiere acelerar los ciclos del capital, hacer girar la rueda de la valorización la mayor cantidad de veces posible; en situaciones de crisis extiende la lógica aceleradora hacia delante por medio del crédito, que produce a su vez sus propias crisis, aceleradas y aceleradoras ellas mismas; el capital tiene además consecuencias desestabilizadoras sobre la cultura, la identidad y la naturaleza del trabajo, amén de los deseos y necesidades de las personas; pone en marcha procesos de cambio tecnológico, que expanden autónomamente el proceso de aceleración social.

Sin embargo, la lógica del capital tiene otra singularidad: su tendencia expansiva respecto a aquello que puede tomar por objeto. No se trata ya de sus consecuencias desestabilizantes sobre aspectos noeconómicos, sino de la lógica económica insertándose en fenómenos no-económicos. El modo convencional de resumir esta tendencia es decir que el capital ve en todo una mercancía, lo que significa que el valor de uso puede apreciarse como valor de cambio, producible en condiciones capitalistas y vendible en un mercado competitivo. Que esto es una tendencia efectiva del capital está fuera de discusión; lo importante, y lo que formulamos como hipótesis ahora, es que se trata de un mecanismo que explica la aceleración en esferas cada vez más amplias de la organización social. Así como la alienación, la conversión del valor de uso en valor de cambio y la explotación vienen juntas con la lógica del capital, y surge con ellas la aceleración en las diversas formas que hemos señalado. Si esto es cierto, tenemos un principio teórico que permite conectar la aceleración básica del capitalismo con la aceleración de cualquier otro aspecto de la organización social.

Solo porque lo tenemos cerca podemos apelar al ámbito académico para ilustrar este mecanismo. La conversión del valor de uso "conocimiento" en el valor de cambio "producto" (típicamente el paper) tiene entre sus consecuencias la reducción del tiempo de producción como imperativo de valorización. En la medida en que rige un tiempo social medio necesario para su producción, hay un incentivo de mínima de alcanzar ese plazo y un incentivo de máxima por reducirlo, acreditando el valor consiguiente en términos de precio. Hacerlo más rápido se vuelve por ello una compulsión estructural que no se impone de modo más enfático por lo difuso de la relación entre valor y precio en un mercado que, como el académico, no es monetario. La ampliación que realiza Bourdieu de la noción de capital puede permitir, en una continuidad del análisis, precisar y apreciar empíricamente esta lógica.

\section{Aceleración y flexibilidad}

Los análisis previos identifican algunos aspectos de la lógica fundamental del capitalismo. Se puede estudiar luego en procesos históricos y sociales concretos el modo en que esa lógica se expresa en realizaciones singulares. Harvey, como dijimos, cumple con la tarea en varias obras, de las cuales nos interesa ahora una (Harvey, 1999) en la que identifica la versión contemporánea de esta lógica general bajo el término genérico de "acumulación flexible". Puede decirse que todas las tendencias aceleradoras que identificamos experimentan con la acumulación flexible una radicalización, y, por ende, hay una sintonía completa entre flexibilidad y aceleración.

Históricamente, la acumulación flexible sucede al régimen fordista de acumulación, caracterizado por la presencia fuerte del Estado y por acuerdos intra y transnacionales que permitieron el ciclo virtuoso de la posguerra hasta los años setenta. Según Harvey, ese sistema de aplazamiento y morigeración de las 
contradicciones entró en crisis para dar lugar a un nuevo régimen de acumulación, al que denomina "flexible" por oposición a las rigideces del modelo previo. Lo flexible es, concretamente, cada uno de los aspectos de la fórmula básica (D -- [FT (cv) + MP (cf)] --- M ---- D'). El capital en su forma dinero (D) se vuelve flexible por el despliegue inédito de las finanzas, que da a la movilidad del dinero en tiempo real una dimensión desconocida. En muchos aspectos la fuerza de trabajo (FT) se ha vuelto flexible, tanto como lo requiere el capital. Es flexible por la tendencia a la pérdida de derechos, la volatilidad de los mercados de trabajo, el incremento de la movilidad territorial de los trabajadores, y la relación cambiante con las formas de trabajo y producción. El capital fijo se ha flexibilizado (MP) también en varias direcciones y sentidos. El desplazamiento de la producción hacia sectores de servicios implica inversiones de más corto plazo en capital fijo; la duración de los medios de producción es tendencialmente menor, siguiendo la lógica de la obsolescencia planificada; el desarrollo tecnológico hace más rápida la reposición. Así mismo, las mercancías producidas $(\mathrm{M})$ son flexibles en el sentido de que se adaptan con más precisión a nichos reducidos de mercado; implican formas de circulación más rápida y eficiente, etcétera.

En la medida en que la flexibilidad significa maleabilidad y adaptación, su consecuencia general es la de facilitar el despliegue de la lógica del capital y sus consecuencias, incluida la aceleración social:

1. En cuanto a la primera forma de aceleración, la de la reducción del tiempo de trabajo (sección 2, punto 1), la flexibilidad de la fuerza de trabajo la facilita de distintas maneras que se resumen en la posibilidad de intensificar y perfeccionar la explotación directa del trabajo.

2. Respecto al ciclo de rotación (sección 2, punto 2), cada una de las tendencias del párrafo anterior le son directamente funcionales. A mayor flexibilidad de cada uno de los pasos, más cerca de pasar el capital de una fase a otra "con la velocidad del pensamiento".

3. La flexibilidad del capital financiero hace también más eficiente su carácter de lubricante temporal (sección 4). A mayor movilidad del capital en tiempo real, mayor eficacia potencial del crédito como expansión hacia adelante, y mayor su capacidad de producir arreglos temporales entre ramas de la producción, producción y consumo, etcétera. También, y de manera más evidente, aumenta la importancia y frecuencia de sus "distorsiones absurdas" y de las crisis del capital ficticio.

4. El cambio tecnológico, la erosión de las formas culturales tradicionales, la afectación de modos de trabajo e identidades laborales, y la producción constante de nuevas necesidades y consumos (sección 5) aumentan todos en volumen e importancia con la flexibilidad. El debilitamiento de la estabilidad cultural, institucional e identitaria también se intensifica.

5. Todos estos procesos hacen más factible técnicamente que la lógica del capital se inserte en esferas más amplias de la vida social (sección 6). Cuanto más maleables son sus componentes, más fácilmente puede el capital penetrar lógicas de acción e interacción previamente extrañas, como lo muestra, entre otros fenómenos, la inserción de la lógica mercantil en el mundo académico en las últimas décadas.

\section{El capital como estructura}

El análisis de Harvey enfatiza en cada punto el carácter contradictorio del capitalismo, lo que implica que sus tendencias no son unívocas ni operan en un vacío social. Todo lo que acabamos de decir tiene que matizarse en este sentido pues nos limitamos a subrayar los aspectos del capitalismo que impulsan la aceleración, sin considerar contrapuntos ni contratendencias. ${ }^{7}$ Por tanto, debe quedar claro que se trata de necesidad en sentido lógico más que empírico, pues son consecuencias de la lógica del capital analizada como tal, en términos abstractos y en una sola de sus dimensiones. Además, debe hacerse lugar a la intervención de los actores, de cuyas capacidades y fuerzas relativas depende en gran parte la configuración histórica de las tendencias. Se puede recordar al respecto el estudio clásico de Thomson (1995) sobre el proceso de imposición 
de la disciplina temporal en los comienzos del capitalismo: muestra allí que se trata justamente de procesos abiertos en su contenido y en parte contingentes respecto a sus resultados.

Con estas aclaraciones podemos volver al argumento de Rosa sobre los "motores" de la aceleración. El análisis que hicimos muestra las razones por las que la competencia y el capitalismo impulsan efectivamente procesos de aceleración social. Lo hacen de varias maneras y en distintos sentidos, siempre por razones discernibles y con rango lógicamente explicativo. Pero el argumento permite también dar sentido a la afirmación de Rosa de que se trata del motor "principal" de la aceleración (Rosa, 2016, p. 46). Siguiendo nuestro análisis, lo es porque la lógica del capital abarca conexiones concretas con los otros motores. El motor cultural de la "promesa de eternidad" se puede derivar de un orden económico que expande la oferta de productos y que necesita producir siempre nuevos deseos y necesidades. La expansión tecnológica es también una necesidad del capital, que en busca de plusvalía relativa pone en marcha y mantiene viva la escalada de innovación. La inestabilidad de las instituciones, normas, hábitos y formas de vida es una consecuencia de la implantación de la lógica del capital y un efecto permanente de su funcionamiento "normal". Y lo mismo puede decirse del incremento del "ritmo de vida", el otro motor que menciona Rosa. Obviamente esto no implica que el motor capitalismo subordine a los demás en el sentido axiomático de cierta tradición marxista. Se trata de mostrar cómo un motor da parte de su impulso también a los otros, lo que no excluye la posibilidad de la relación inversa.

$\mathrm{Al}$ analizar el capitalismo en términos de lógica del capital, aportamos al argumento de Rosa algo adicional: la tematización de los motores en términos estructurales. La idea de estructura social, como otras importantes de la tradición sociológica, está tenuemente presente en el argumento de Rosa. ${ }^{8}$ Se puede interpretar que el motor "capitalismo" es estructural en un sentido preciso del término, según el cual es estructural lo esencial de una configuración social, lo que tiene permanencia en el tiempo, lo que tiene un orden o una lógica propia, y muy especialmente lo que opera con independencia relativa de la consciencia y voluntad de los actores (Cristiano, 2017). Todos estos rasgos son característicos de la lógica del capital. Es esencial al orden capitalista en el sentido de que no podemos hablar de capitalismo si no está presente (sección 2), misma razón por la cual es lo que permanece en el tiempo, adoptando distintas configuraciones pero con continuidad esencial. Además, es una lógica, un proceso discernible en sus razones y comprensible en la dirección de su desarrollo, y es sin duda un autónomo respecto a la consciencia y voluntad de los actores en el sentido de que impone sus reglas sin necesidad de que ellos las reconozcan como tales.

Pensar como estructural la lógica del capital tiene una doble ventaja analítica. Permite conectar el núcleo estructural con fenómenos estructurales secundarios y con fenómenos no estructurales sino coyunturales. La propuesta de Rosa ha generado a veces incomodidad por su trazo excesivamente amplio y por su contraste con registros empíricos más locales y acotados. ${ }^{9}$ Situar como estructural la lógica de la aceleración implica reconocer que hay otros niveles estructurales, que hay planos coyunturales que se derivan de la estructura, y que hay numerosas mediaciones entre el núcleo estructural y aspectos más específicos de la organización social. Idealmente, un concepto explícito de estructura permite apreciar estas mediaciones, vinculando una tendencia general discernible (la aceleración en la lógica del capital) con las realidades empíricas que la expresan, procesan y resisten.

El segundo interés tiene que ver con el par conceptual que se asocia convencionalmente a estructura, que es el de acción. Dijimos que de la acción y la fuerza relativa de los actores depende en gran medida el modo en que la lógica básica se despliega en situaciones particulares. Por ejemplo, la imposición de la disciplina horaria en el taller fue incorporada al ethos de los trabajadores en un largo proceso de luchas, que se reproducen luego en el capitalismo periférico o en sectores de la producción alcanzados menos directamente por esa lógica. Esto significa que la afirmación de que la tendencia aceleradora es estructural no implica que sea inexorable en su forma, ritmo o contenido, puesto que todo depende del modo en que los actores se adapten o luchen en relación con ella. 
Lo anterior nos deja por último en el punto al que el propio Rosa quiere llevar su argumento, que es el de la resistencia y la contestación a la "tiranía de la aceleración". Su intención es rehabilitar un modo de teoría crítica que parte de las experiencias vividas por los actores, las cuales, en este caso, son de sufrimiento pasivo por un orden temporal que no comprenden, y que les impone costos psicológicos, identitarios y políticos. El primer requisito de cualquier contestación, y lo que puntualmente puede aportar la investigación social, es claridad respecto a las causas, aportando sentido al sufrimiento subjetivo. Pero además esas experiencias pueden ser muy diversas, abarcar de manera parcial y diferenciada a los distintos grupos sociales, y por ende estar lejos de un padecimiento unánime y uniforme. También a esto contribuye el procesamiento del análisis en términos estructurales, pues permite apreciar los mecanismos que irradian la tendencia estructural en distintas direcciones y con diferentes intensidades.

\section{Referencias}

Blondeau, O., Whiteford, N. D., Vercellone, C., Kyrou, A., Corsani, A., Rullani, E., Moulier Boutang, Y., y Lazzarato, M. (2004). Capitalismo cognitivo, propiedad intelectual y creación colectiva. Madrid: Traficantes de Sueños.

Braverman, H. (1987). Trabajo y capital monopolista. La degradación del trabajo en el siglo XX. Ciudad de México: Nuestro Tiempo.

Campos Medina, F., y Campos Medina, L. (2016). Estructuras, regímenes y arreglos espacio-temporales: diálogos con la teoría de la aceleración de Hartmut Rosa. Acta Sociológica, (69), 77-108.

Castree, N. (2009). The spatio-temporality of capitalism. Time \& Society, 18(1), 26-61.

Cristiano, J. (2017). Contribución al desarrollo de una teoría abstracta de la estructura social. Miríada, 9(13).

Harvey, D. (1991). Los limites del capitalismo y la teoría marxista. Ciudad de México: Siglo XXI Editores.

Harvey, D. (1999). La condición de la postmodernidad. Buenos Aires: Amorrortu.

Harvey, D. (2007). Espacios del capital. Madrid: Akal.

Harvey, D. (2014). Diecisiete contradicciones y el fin del capitalismo. Madrid: Traficantes de Sueños.

Marx, K. (1988). Manifiesto del Partido Comunista. Buenos Aires: Centro Editor de Cultura.

Melucci, A. (1998). Inner time and social time in a world of incertainty. Time \& Society, 7(2), 179-191.

Postone, M. (2005). Repensando a Marx (en un mundo post marxista). En Lo que el trabajo esconde (pp. 249-283). Madrid: Traficantes de Sueños.

Postone, M. (2006). Tiempo, trabajo y dominación. Madrid: Marcial Pons.

Ramos Torre, R. (2014). Atemporalización y presentificación del mundo social en la sociología contemporánea. Politica y Sociedad, 51(1), 147-176.

Revista Acta Sociológica, 69 (2016). http://www.revistas.unam.mx/index.php/ras/issue/view/Acta\%20Sociol\%C3\% B3gica\%2069

Rosa, H. (2003). Social acceleration: ethical and political consequences of a desynchronized high-speed society. Revista Constellations, 10(1), 3-33. https://onlinelibrary.wiley.com/toc/14678675/2003/10/1

Rosa, H. (2011). Aceleración social. Consecuencias éticas y políticas de una sociedad de alta velocidad desincronizada. Persona y Sociedad, XXV(1), 9-49.

Rosa, H. (2013). Social acceleration. A new theory of modernity. New York: Columbia University Press.

Rosa, H. (2016). Alienación y aceleración. Hacia una teoria critica de la temporalidad en la modernidad tardía. Buenos Aires: Katz.

Rosa, H., y Scheuerman, W. (eds.). (2009). High-spead society: social aceleration, power and modernity. Pennsylvania: The Pennsylvania State University Press.

Sennet, R. (2006). La corrosión del carácter. Las consecuencias personales del trabajo en el nuevo capitalismo. Barcelona: Anagrama. 
Thompson, E. (1995). Tiempo, disciplina de trabajo y capitalismo industrial. En Costumbres en común (pp. 395-452). Barcelona: Crítica.

Tietze, S., y Musson, G. (2002). When “works" meets "home”. Temporal flexibility as lived experience. Time \& Society, $11(2-3), 315-334$.

Torres, E. (2016). Reseña de Social Aceleration, de H. Rosa. Política y Sociedad, XXX(2), 121-130.

Vostal, P. (2014). Tematizing speed: between critical theory and cultural analysis. European Journal of Social Theory, $17(1), 95-114$.

\section{Notas}

* Artículo de reflexión enmarcado en el proyecto PICT 2015-1824 de la ANCyT (MinCyT, Argentina)

1 La principal referencia es Rosa (2013), que tiene versiones resumidas en Rosa (2016) y Rosa (2011). Véase también Rosa y Scheuerman (2009). Como introducción al debate generado, puede verse el temprano monográfico de Constellations (vol. 10, n. ${ }^{\circ} 1$ del 2003) y el más reciente en castellano de Acta Sociológica (vol. 69 del 2016). La revista Time \& Society ha retomado en diversos artículos de los últimos años las tesis de Rosa. El trabajo de Vostal (2014) pone la propuesta de Rosa en la perspectiva más amplia de las discusiones sobre aceleración y velocidad en la teoría social reciente.

2 Véase el estado de la cuestión presentado por Castree (2009, pp. 29-32). La obra de Harvey es conocida sobre todo por sus aportes a la geografía y los estudios urbanos, y alcanzó amplio reconocimiento con La condición de la postmodernidad a fines de los noventa. El libro que el propio Harvey considera esencial, y el que tomamos como referencia principal en este artículo, es sin embargo The Limits of Capital (Harvey, 1991), base de todos sus trabajos posteriores. Dice el propio Harvey que, aun así, es su libro menos leído (Harvey, 2007, p. 21), afirmación que puede suscribirse en lo que respecta a la circulación de la obra en el mundo de habla hispana. Hartmut Rosa hace varias alusiones a Harvey en su análisis del motor "capitalismo" (Rosa, 2013, pp. 161-174; 2011, p. 23) pero, con una sola excepción (2013, p. 218), no toma como referencia ese libro. Además de Harvey, en el intento de leer críticamente el capitalismo en perspectiva temporal, destaca el libro de Postone (2006).

3 La lectura de Postone antes mencionada radicaliza esta conclusión. A su juicio, el valor es el corazón del análisis de Marx, más incluso que la explotación y la división de clases, por lo que el tiempo es la compulsión estructural más importante del capitalismo. Cfr. Postone (2006, cap. 5) y Postone (2005, pp. 265-272).

4 Para un amplio panorama, véase Ramos Torre (2014).

5 Véase a modo de ejemplo Sennet (2006), Melucci (1998), y Tietze y Musson (2002).

6 Véase, por ejemplo, el trabajo clásico de Braverman (1987) y más recientemente la discusión promovida por la perspectiva del "capitalismo cognitivo" (Blondeau et al., 2004).

7 Por ejemplo, el capitalismo crea barreras contra la expansión ilimitada del cambio tecnológico (Harvey, 1991, pp. 140-141), lo mismo que existe un factor de inercia en las inversiones a largo plazo (p. 96).

8 El principal empleo que hace Rosa de la idea de estructura proviene de Luhmann. A su juicio, la aceleración "del cambio social" surge de la diferenciación sistémica como fenómeno estructural, puesto que implica una multiplicación de opciones y la consiguiente necesidad de acelerar procesos de selección. Se podría con el mismo criterio considerar estructural al motor económico del capitalismo, pero no es la opción de Rosa. Sobre el significado y las tensiones que produce en Rosa esta operación véase Torres (2016, pp. 127-129).

9 Véase, por ejemplo, la crítica de Campos Medina y Campos Medina (2016) y la de Ramos Torre (2014, pp. 165-166), dirigidas más ampliamente a las posturas que unifican tendencias temporales.

\section{Licencia Creative Commons CC BY 4.0}

Cómo citar este artículo: Cristiano, J. L. (2019). La aceleración en la lógica del capital. Universitas Humanistica, 88. https://doi.org/10.11144/Javeriana.uh88.lalc 